\title{
Property S[a,b]: A Direct Approach
}

\author{
George Nickolaos Miliaras \\ American University of Athens, Athens, Greece \\ E-mail:gmiliara@yahoo.gr,gmiliaras@aua.edu \\ Received May 18, 2011; revised June 17, 2011; accepted June 30, 2011
}

\begin{abstract}
In this paper we prove directly that the property $S[a, b]$, implies $[a, b)$-compact, and under certain conditions it implies $[a, b]$-compact.
\end{abstract}

Keywords: Compactness number, $[a, b]$-compact, $[a, b)$-compact, property $S[a, b]$

\section{Introduction}

Compactness is one of the oldest and the most famous notions in mathematical analysis and especially in topology. A partial generalization is $[a, b]$-compactness [1-8]. This has been shaped to property $S[a, b]$ by Vaughan in 1975 [8], (page 253 and 256-257) who proved that $[a, b]$-compactness is equivalent to $S[a, b]$ if $c f(b) \geq a$ (Theorem 2C) using the Corollary of Lemma 2 (pages 254-255).

In this paper we are going to prove directly something stronger, which we will need the following definitions:

Definition 1. The compactness number $C n(X)$ of a space $X$ is the least cardinal $k$ such that every open cover of $X$ has a subcover of cardinality less than $k$.

Definition 2. A space $X$ is called $[a, b]$-compact ([a,b)-compact) if every open cover $U$ of $X$ with $|U| \leq b \quad(|U|<b)$ has a subcover of cardinality strictly less than $a$.

Definition 3. A space $X$ is said to have property $S[a, b]$ if every open cover of $X$ of regular cardinality less than $b$, has a subcover of cardinality strictly less than a.

\section{Main Result}

Theorem. Let $X$ have the property $S[a, b]$, then $X$ is $[a, b)$-compact, and if $b$ is regular or if $c f(b) \geq a$, then $X$ is $[a, b]$-compact, furthermore either $C n(X)>b$, or, $a=b=C n(X)$.

Proof. We study the following three cases:

Case 1. $C n(X)>b$
Let $U_{*}$ be an open cover of $X$ with $\left|U_{*}\right|=k$ be the first singular cardinal greater than $a$, with the property that $U_{*}$ has no subcover with cardinality less than $a$.

If $k>b$, then clearly $X$ is $[a, b]$-compact.

If $k=b$, then clearly $X$ is $[a, b)$-compact.

Assume that $k<b$. Since $k<b<C n(X)$, there exists an open cover $V_{*}$ of $X$ which $\left|V_{*}\right|=\lambda>k$. Then at least one $U \in U_{*}$ is covered by a collection $V_{*}^{\prime} \subset V_{*}$ with $\left|V_{*}^{\prime}\right|=\mu>k$, such that no subcollection of $V_{*}^{\prime}$ with less than $\mu$ elements can cover $U$. If such a $U$ didn't exist it would contradict the hypothesis $C n(X)>b>k$. Consider the collection of open sets $W_{*}=\left\{V_{\gamma} \cap U \mid V_{\gamma} \in V_{*}^{\prime}\right\}$. Let a collection $W_{*}^{\prime}=\left\{W_{\gamma} \mid \gamma \in k^{+}\right\}$of $k^{+}$elements of $W_{*}$ and let $W$ be the union of the rest of the remaining elements of $W_{*}$ (if there are any left). We have $U=W \cup\left(\cup W_{*}^{\prime}\right)$, put $U_{*}^{\prime}=U_{*}-\{U\}$, then $S_{*}=U_{*}^{\prime} \cup W_{*}^{\prime} \cup\{W\}$ is an open cover of $X$ with $\left|S_{*}\right|=k^{+}$since $\left|U_{*}^{\prime}\right|=k$, $\left|W_{*}^{\prime}\right|=k^{+}$and $|\{W\}|=1$, then $S_{*}$ has a subcover with cardinality less than $a$, since $k^{+} \leq b$ is a regular cardinal and $X$ has property $S[a, b]$. Now, since $S_{*}$ refines $U_{*}, U_{*}$ must have a subcover with cardinality less than $a$, thus $X$ is $[a, b)$-compact.

Now, if $b$ is a regular cardinal then clearly $X$ is $[a, b]$-compact, since it is $[a, b)$-compact, and has property $S[a, b]$.

Assume that $b$ is singular and $c f(b) \geq a$. Let $U_{*}=\left\{U_{\gamma} \mid \gamma<b\right\}$, be an open cover of $X$, with $\left|U_{*}\right|=b$, let $c f(b)=k \geq a$, choose cardinals $b_{\beta}$, $\beta<k$ with $\sup \left\{b_{\beta}\right\}=b$. For every $\beta<k$, let $V_{\beta}=\cup\left\{U_{\gamma} \mid \gamma<b_{\beta}\right\}$, and let $V_{*}=\left\{V_{\beta} \mid \beta<k\right\}$. Then $V_{*}$ has a subcover $V_{*}$ with $\left|V_{*}\right|=\mu<a$, since $k$ is regular, $k \geq a$ and $X$ has property $S[a, b]$. Let 
$V_{*}^{\prime}=\left\{V_{\beta} \mid \beta<\mu\right\}$, then $V_{\mu+1}=X$, but

$V_{\mu+1}=\cup\left\{U_{\gamma} \mid \gamma<b_{\mu+1}\right\}$. Put $U_{*}^{\prime}=\left\{U_{\gamma} \mid \gamma<b_{\mu+1}\right\}$, then $\left|U_{*}^{\prime}\right|=\left|b_{\mu+1}\right|<b$, then since $X$ is $[a, b)$-compact, if $U_{*}^{\prime} \geq a$, it has a subcover $U_{*}^{\prime}$ with $\left|U_{*}^{\prime}\right|<a$, thus $X$ is $[a, b]$-compact.

Case 2. $\operatorname{Cn}(X)=b$

Assume that $b$ is a limit cardinal. Since $X$ has property $S[a, b]$, for every $\lambda<b, X$ has property $S[a, \lambda]$, and therefore $X$ is $[a, \lambda)$-compact for every $\lambda<b$, and if $\lambda$ is regular or $c f(\lambda) \geq a, X$ is $[a, \lambda]$-compact, by case 1 . Let $U_{*}$ be an open cover of $X$, since $C n(X)=b, U_{*}$ has a subcover $U_{*}^{\prime}$ such that $\left|U_{*}^{\prime}\right|=k<b$. Now, since $b$ is a limit cardinal, $k^{+}<b$, it follows from the above that $X$ is $\left[a, k^{+}\right]$-compact, so $U_{*}^{\prime}$ has a subcover $U_{*}^{\prime \prime}$ such that $\left|U_{*}^{\prime \prime}\right|<a$, thus $X$ is $[a, b]$-compact and since $C n(X)=b$, we must have $a=b=C n(X)$. Assume that $b$ is a successor cardinal $k^{+}$, then $X$ has property $S\left[a, k^{+}\right]$, therefore $X$ has property $S[a, k]$ and therefore $X$ is $[a, k)$-compact. If $k$ is regular or $c f(k) \geq a, X$ is $[a, k]$-compact, by case 1 , and since $C n(X)=k^{+}, X$ is $\left[a, k^{+}\right]$-compact, thus $X$ is $[a, b]$-compact, and since $\operatorname{Cn}(X)=b$ we have $a=b=C n(X)$.

Assume that $c f(k)<a$. Let $U_{*}$ be an open cover of $X$ with $\left|U_{*}\right|=k$, with no subcover with cardinality less than $k$. Let $V_{*}$ be an open cover of $X$ with $\left|V_{*}\right|=k^{+}$. Consider the open cover

$W_{*}=\left\{W=U \cap V \mid U \in U_{*}, V \in V_{*}\right\}$. Then $\left|W_{*}\right|=k^{+}$and also $W_{*}$ refines $U_{*}$, since $X$ has property $S\left[a, k^{+}\right], \quad W_{*}$ has a subcover $W_{*}^{\prime}$ with $\left|W_{*}^{\prime}\right|<a$ and since $W_{*}^{\prime}$ refines $U_{*}, U_{*}$ has a subcover $U_{*}^{\prime}$ with $\left|U_{*}^{\prime}\right|<a$, thus $X$ is $[a, k]$-compact and since $C n(X)=k^{+}=b, X$ is $[a, b]$-compact and using the previous argument $a=b=C n(X)$.
Case 3. $C n(X)<b$

Let $C n(X)=\lambda<b$, then $X$ has property $S[a, \lambda]$, so $C n(X)=\lambda=b=a$ by case 2 . Now since

$C n(X)=a, X$ is $[a, c]$-compact for every $c \geq a$, therefore $X$ is $[a, b]$-compact.

The proof is complete.

\section{References}

[1] P. S. Alexandroff and P. Urysohn, "Memorie sub les Espaces Topologiques Compacts," Koninklijke Akademie van Wetenschappen, Amsterdam, Vol. 14, 1929, pp. 1-96.

[2] R. E. Hodel and J. E. Vaughan, “A Note on [a,b] Compactness," General Topology and Its Applications, Vol. 4, No. 2, 1974, pp. 179-189.

doi:10.1016/0016-660X(74)90020-8

[3] G. Miliaras, "Cardinal Invariants and Covering Properties in Topolology," Thesis, Iowa State University, Amsterdam, 1988.

[4] G. Miliaras, "Initially Compact and Related Spaces," Periodica Mathematica Hungarica, Vol. 24, No. 3, 1992, pp. 135-141. doi:10.1007/BF02330872

[5] G. Miliaras, "A Review in the Generalized Notian of Compactness,” Unione Matematica Italiana, Vol. 7, 1994, pp. 263-270.

[6] G. Miliaras and D. E. Sanderson, "Complementary Forms of $[\alpha, \beta]$-Compact," Topology and Its Applications, Vol. 63, No. 1, 1995, pp. 1-19. doi:10.1016/0166-8641(95)90001-2

[7] R. M. Stephenson, "Initially k-Compact and Related Spaces,” In: K. Kuuen and J. E. Vaughan, Eds., Handbook of Set-Theoritic Topology, North-Holland, Amsterdam, 1984, pp. 603-632.

[8] J. E. Vaughan (Greensbaro N. C.), "Some Properties Related to [a,b]-Compactness," Fundamenta Mathematicae, Vol. 87, 1975, pp. 251-260. 